\title{
PLANTS OF THE LAST MOUNTAIN LAKE NATIONAL WILDLIFE AREA
}

J.R. CALDWELL, P.S. TAYLOR and E.A. DRIVER, Canadian Wildlife Service, 115 Perimeter Road, Saskatoon, Saskatchewan, S7N OX4 and L.J SHANDRUK, Canadian Wildlife Service, 4999-98 Avenue, Edmonton, Alberta. T6B 2 X3

The Last Mountain Lake National Wildlife Area, located at the northern end of Last Mountain Lake, is within the Mixedgrass Prairie Ecodistrict of the Grasslands Ecoregion. ${ }^{3}$ In Canada most of this grassland type has been drastically altered by agricultural practices including cultivation and intensive grazing. Thus, few areas have maintained their native character. The habitats within the wildlife area include grasslands which have never been subjected to intensive agricultural practices and remain in nearly their natural state.

This preliminary annotated plant list is based largely upon collections housed in the Canadian Wildlife Service Herbarium, Saskatoon. Most of the specimens were collected by G.G. Anweiler in 1969 and P.S. Burton in 1983, but W.C. Harris, E.A. Driver, P.S. Taylor and J.R. Caldwell also contributed with their findings. This list is not a complete flora, for the wildlife area has not been thoroughly searched for plants.

This annotated list includes 318 species and one variety found in or near the Last Mountain Lake National Wildlife Area. The list is organized into 58 plant families arranged in the order accepted by most standard manuals. ${ }^{6}$ Information on habitat was obtained from herbarium collection labels. The scientific and common names used follow those of Moss where possible. $^{8}$

Most of the species listed are common on the National Wildlife Area, but a few are uncommon, including Water Foxtail,

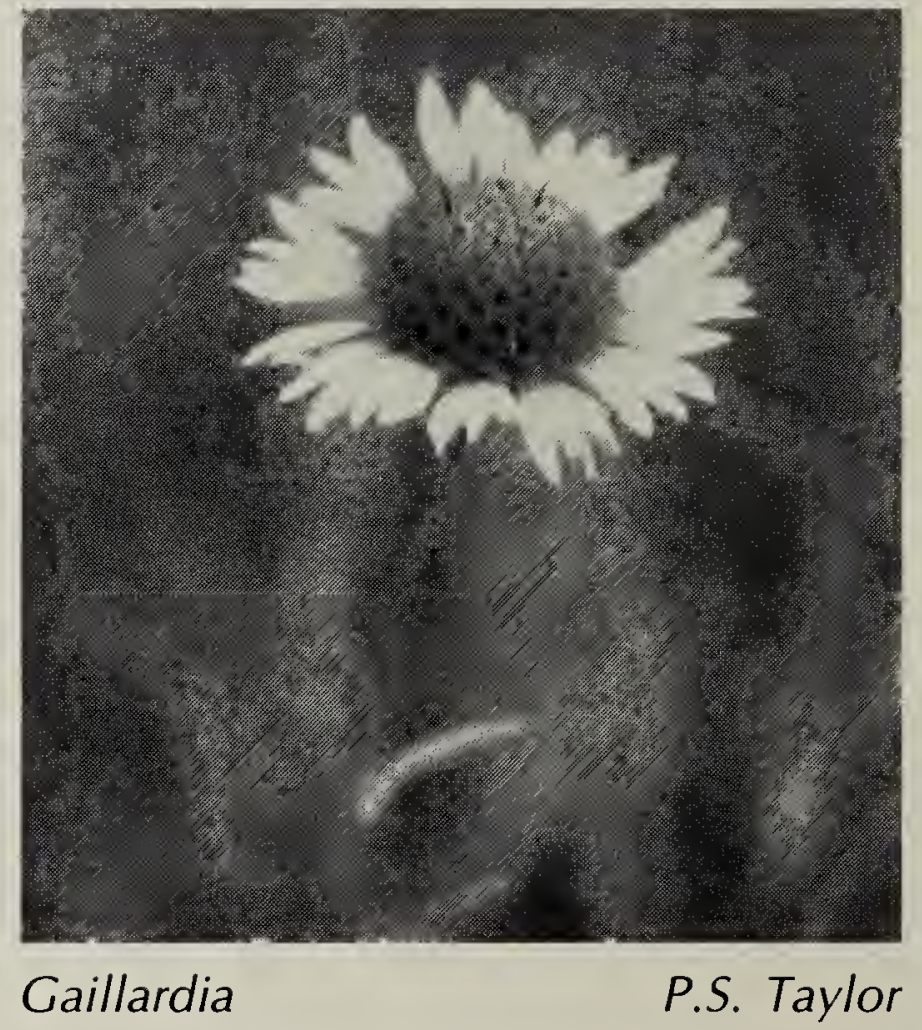

Sheep Fescue, Salt-marsh Sand Spurry, Few-flowered Aster and Upland White Goldenrod. The provincial floral emblem, the Western Wood Lily, was found in several locations, but is not abundant.

Because all plants are protected in the National Wildlife Area, observations of new or unusual plants should be documented by taking careful notes and photographs. Such information would be welcomed by the Area Manager or Habitat Management staff in Saskatoon.

\section{Acknowledgements}

The authors would like to thank J.Y. Marchand and V.L. Harms of the Fraser Herbarium, University of Saskatchewan, Saskatoon, for their assistance in verifying the identity of plants collected at Last Mountain Lake. 
1 BAILEY, L.H. 1954. Manual of cultivated plants. Revised Edition. MacMillan Company, New York.

2 FERNALD, M.L. 1950. Gray's manual of botany. Eighth Edition. Am. Book Co.

3 HARRIS, W.C., A. KABZEMS, A.L. KOSOWAN, G.A. PADBURY and J.S. ROWE 1983. Ecological regions of Saskatchewan. Sask.Parks and Renewable Res. Tech. Bull: No. 10.

4 HUDSON, J.H. 1977. Carex in Saskatchewan. Bison Publ. House, Sask.

5 KARTESZ, J.T. and R. KARTESZ 1980. A synonymized checklist of the vascular flora of the United States, Canada and Greenland. Vol II. The Biota of North America. The Univ. of North Carolina Press, USA.
6 LOOMAN, J. and K.F. BEST 1979. Budd's flora of the Canadian prairie provinces. Agric. Canada Publ. 1662, Ottawa.

7 MAHER, R.V., G.W. ARGUS, V.L. HARMS and J.H. HUDSON 1979. The rare vascular plants of Saskatchewan. Syllogeus Series, No 20. National Mus. of Nat. Science. Ottawa.

8 MOSS, E.H. 1983. Flora of Alberta. Second Edition, Univ. of Toronto Press, Toronto.

9 SCOGGAN, H.J. 1957. Flora of Manitoba. National Mus. of Canada, Bull. No. 140, Biol. Ser., No. 47, Dept. of Northern Affairs and Nat. Res., Ottawa.

10 VANCE, F.R., J.R. JOWSEY and J.S. MCLEAN 1984. Wildflowers across the prairies. Second Ed. Western Producer Prairie Books, Saskatoon.

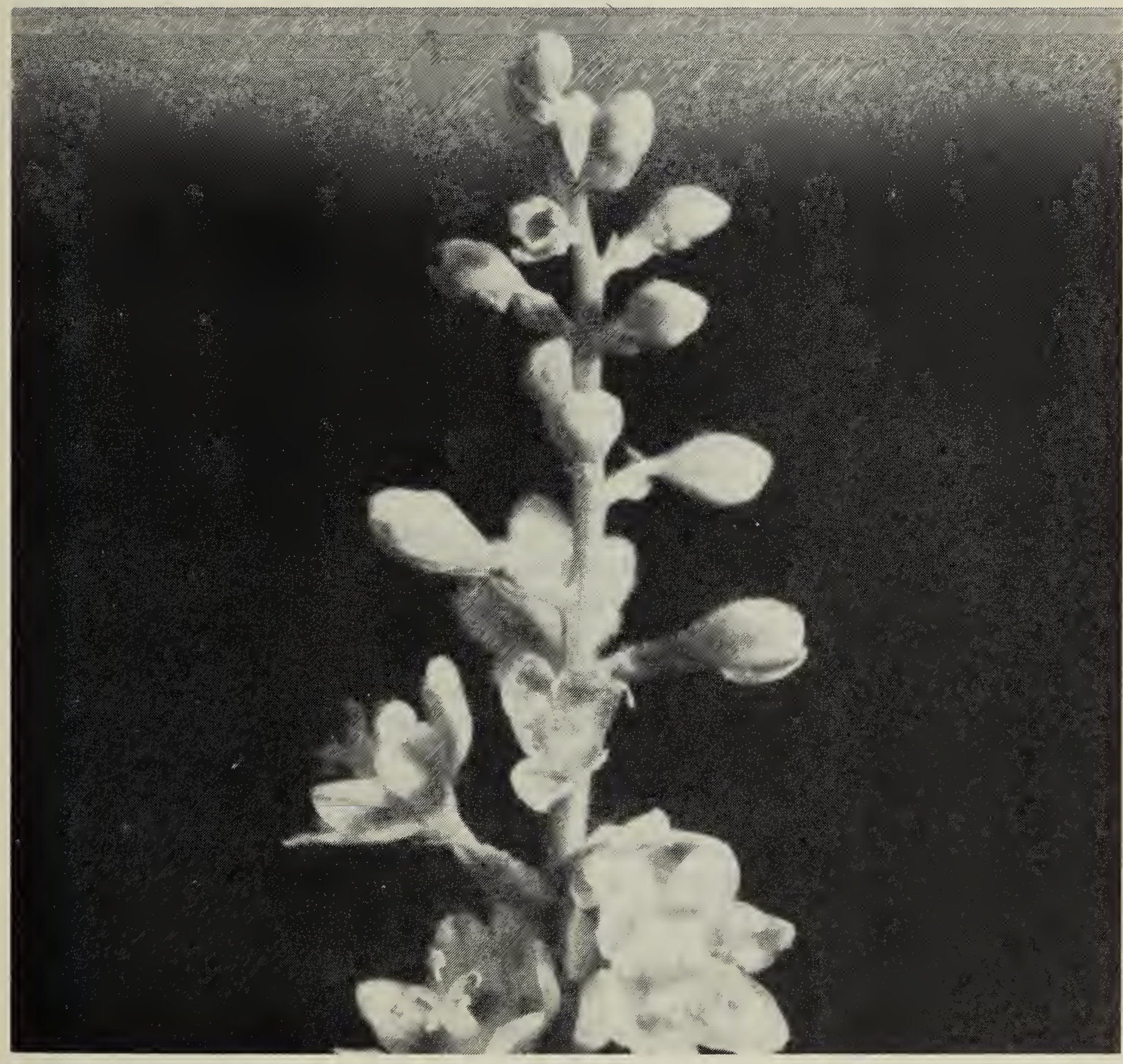


ANNOTATED LIST OF VASCULAR PLANTS OF LAST MOUNTAIN LAKE AND STALWART MARSH NATIONAL WILDLIFE AREAS

\section{COMMON NAME \\ SPECIES}

Spike-moss Family: Selaginellaceae

Prairie Selaginella Selaginella densa Rydb.

\section{Horsetail Family: Equisetaceae}

Common Scouring-rush

Smooth Scouring-rush

Meadow Horsetail

\section{Cattail Family: Typhaceae}

Common Cattail

Equisetum hyemale $\mathrm{L}$.

Equisetum laevigatum A.Br.

Equisetum pratense Ehrh.

\section{HABITAT}

grasslands, dry soils, eroded sites

wetland margins, low areas sandy lake shore, moist areas moist sandy areas

slough margins, marshes, lakes

ponds, marshes, lakes

Potamogeton pectinatus $\mathrm{L}$.

Potamogeton richardsonii

(Benn.) Rydb.

ponds, marshes, lakes

Potamogeton vaginatus Turcz. sloughs, lake, Basin A

Sheathed Pondweed
Widgeon Grass W. Ditch-grass Ruppia occidentalis Wats.

\section{Arrow-grass Family: Juncaginaceae}

Seaside Arrow-grass

Marsh Arrow-grass
Triglochin maritima L.

Triglochin palustris $\mathrm{L}$. wet areas, near lake

marshy areas

\section{Water-plantain Family: Alismaceae}

Narrow-leaved Water-plantain Alisma gramineum K.C. Gmel.sloughs, marshes

Common Water-plantain

Arum-leaved Arrowhead

\section{Grass Family: Gramineae}

Crested Wheatgrass

Northern Wheatgrass

Quack Grass

Western Wheatgrass

Slender Wheatgrass

Rough Hair Grass

Water Foxtail

Wild Oat

Slough Grass

\section{Blue Grama}

Awnless/Smooth Brome

Northern Reed Grass

Plains Reed Grass

Narrow Reed Grass

Sand Grass

Salt Grass

Canadian Wild Rye Grass
Alisma plantago-aquatica L.

Sagittaria cuneata Sheld.

Agropyron cristatum (L.)

Gaertn.

Agropyron dasystachyum

(Hook.) Scribn.

Agropyron repens (L.) Beauv.

Agropyron smithii Rydb.

Agropyron trachycaulum

(Link) Malte

Agrostis scabra Willd.

Alopecurus geniculatus L.

Avena fatua $\mathrm{L}$.

Beckmannia syzigachne

(Steud.) Fern.

sloughs, marshes

slough edges

Bouteloua gracilis (HBK.) Lag. dry native grassland

Bromus inermis Leyss.

Calamagrostis inexpansa

A.Gray

Calamagrostis montanensis

Scribn.

Calamagrostis neglecta (Ehrh.)

Gaertn., Mey. \& Schreb. wet meadows, slough edges

Calamovilfa longifolia (Hook.)

Scribn.

Distichlis stricta (Torr.) Rydb. saline flats

Elymus canadensis L. sandy areas

seeded for pasture and hay

native grasslands

distured areas, introduced moist areas, prairie, parkland moist areas around lakes and sloughs

meadows, woods, waste places moist soils, slough edges

croplands, introduced

slough margins, wet areas

roadsides, seeded pastures

wet meadow, on island

moist grasslands

beaches, banks, sandy areas 
COMMON NAME

Northern Rough Fescue

Sheep Fescue

Tall Manna Grass

Hooker's Oat Grass

Sweet Grass

Wild Barley

Barley

June Grass

Prairie Muhly

Mat Muhly

Witch Grass

Common Reed Grass

Canby Blue Grass

Early Blue Grass

Fowl Blue Grass

Kentucky Blue Grass

Nuttalls' Salt-meadow Grass

Spangletop

Green Foxtail

Alkali Cord Grass

Spear Grass

Western Porcupine Grass

Green Needle Grass

\section{Sedge Family: Cyperaceae}

Awned Sedge

Woolly Sedge

Blunt Sedge

Graceful Sedge

Low Sedge

White-scaled Sedge

Creeping Spike-rush

Tall Cotton-grass

Viscid Great Bulrush

Three-square Bulrush

Prairie Bulrush

Great Bulrush
SPECIES

Festuca altaica Trin.ssp.

hallii(Vasey)Harms

Festuca ovina L.

Glyceria grandis S. Wats.

Helictotrichon hookeri

(Scribn.) Henr.

Hierochloe odorata (L.) Beauv. wet areas, moist grasslands

Hordeum jubatum L.

Hordeum vulgare $\mathrm{L}$.

Koleria gracilis Pers.

Muhlenbergia cuspidata (Torr.)

Rydb.

Muhlenbergia richardsonis

(Trin.) Rydb.

Panicum capillare L.

Phragmites communis Trin.

Poa canbyi (Scribn.) Piper

Poa cusickii Vasey

Poa palustris L.

Poa pratensis L.

Puccinellia nuttalliana

(Schultes) Hitchc.

Scolochloa festucacea (Willd.)

Link

Setaria viridis (L.) Beauv.

Spartina gracilis Trin.

Stipa comata Trin. \& Rupr.

Stipa spartea Trin. var.

curtiseta Hitchc.

Stipa viridula Trin.

Carex atherodes Spreng.

Carex lanuginosa Michx.

Carex obtusata Lilj.

Carex praegracilis W. Boott

Carex stenophylla Wahl.

Carex xerantica Bailey

Eleocharis palustris (L.) R.\& S. shallow wetlands, mud flats

Eriophorum angustifolium

Honck.

Scirpus acutus Muhl.

Scirpus americanus Pers.

Scirpus paludosus Nels.

Scirpus validus Vahl.

\section{HABITAT}

native prairie

moist areas on prairies

slough edges, lakeshore

moist to moderately dry

prairie

saline flats, marsh, roads

lure crops

prairie grasslands

native grasslands

moist, often saline grasslands dry marsh, prairie, introduced lakeshore, marshes

moist, often alkali, meadows dry prairie

moist meadows, lakeshore

pastures, moist prairie

moist to dry alkaline soils

sloughs and marshes

introduced, farmyards, fields

alkali areas, sandy soils

dry prairie

dry prairie

dry to moist shrubby areas

marsh and slough margins

slough margins

dry to moist grasslands

wet meadows, slough edges

dry grasslands

in willow groves

bogs, marshes

lake edge, marshes

marshes, often saline wetlands saline sloughs and marshes sloughs and marshes

ponds, marshes

Lemna minor L.

Juncus balticus Willd. Juncus bufonius L.

shores, sandhills

bog area (springs)
Lily Family: Liliaceae

Pink-flowered Onion
Allium stellatum Fraser native prairie 
COMMON NAME

Prairie Onion

Asparagus

Western Wood Lily

Star-flowered Solomon's-Seal

Smooth Camas

Iris Family: Iridaceae

Blue-eyed Grass

Orchid Family: Orchidaceae

Northern Green Orchid

Willow Family: Salicaceae

Balsam Poplar

Aspen

White Willow

Beaked Willow

Short-capsuled Willow

Sandbar Willow

Basket Willow

Elm Family: Ulmaceae

American Elm

Nettle Family: Urticaceae

Stinging Nettle
SPECIES

HABITAT

Allium textile Nels. \& Macbr. dry prairie

Asparagus officinalis L.

Lilium philalelphicum L.

Smilacina stellata L.

Zyadenus elegans Pursh

Sisyrinchium montanum

Greene

meadows, moist areas

Habenaria hyperborea (L.)

R.Br.

dugout edge near lake

Populus balsamifera L.

Populus tremuloides Michx.

Salix alba L.

Salix bebbiana Sarg.

Salix brachycarpa Nutt.

Salix exigua Nutt.

Salix petiolaris J.E. 'Smith

Ulmus americana L.

shelterbelts, introduced

island, near lakeshor depressions in dry prairie shelterbelts, introduced slough and lake margins wet areas, sand dunes lakeshore, creeks, sloughs near spring

prainie, moist areas, mead prairie, moist areas, meadows moist areas, meadows, woods moist areas, saline meadows

moist areas, in shrubs

\section{Sandalwood Family: Santalaceae}

Bastard Toadflax

Comandra umbellata (L.) Nutt. dry grasslands var. pallida (DC) Jones

Comandra umbellata (L.) Nutt. var. umbellata

prairie grasslands

\section{Buckwheat Family: Polygonaceae}

Yellow Umbrellaplant

Swamp Persicaria

Doorweed

Water Smartweed

Pale Persicaria

Rhubarb

Golden Dock

Field Dock

Narrow-leaved Dock
Eriogonum flavum Nutt. dry prairie, eroded hillsides

Polygonum amphibium L. var. sloughs, lakeshore, mud flats stipulaceum (Coleman) Fern.

Polygonum aviculare L. yards, roadsides, waste places Polygonum coccineum Muhl. var. pratincola (Greene) Stanford Polygonum lapathifolium L. lakeshore, slough margins Rheum rhaponticum L. old farmsteads, introduced Rumex maritimus L. low prairie, saline areas

Rumex pseudonatronatus Borbas Rumex triangulivalvis (Dans.) Rech.f. moist sandy soils

marsh, wet areas

moist, open ground

\section{Goosefoot Family: Chenopodiaceae}

Scurfless Salt-brush

Nuttall's Atriplex

Orache
Atriplex dioica (Nutt.) Macbr. saline flats, eroded clay slopes Atriplex nuttallii S.Wats. Atriplex patula L. dry prairie, eroded soils

roadside, saline meadows 
COMMON NAME

Russian Pigweed

Lamb's-Quarters

Narrow-leaved Goosefoot

Oak-leaved Goosefoot

Winterfat

Summer-cypress

Red Samphire

Russian-thistle

Western Sea-blite

\section{SPECIES}

Axyris amaranthoides $\mathrm{L}$.

Chenopodium album L.

Chenopodium leptophyllum

Nutt.

Chenopodium salinum Standl. saline flats

Eurotia lanata (Pursh.) Moq.

Kochia scoparia (L.) Schrad.

Salicornia rubra A.Nels.

Salsola kali L.

Suaeda depressa (Pursh)

S.Wats.

Amaranth Family: Amaranthaceae

Red-root Pigweed

Amaranthus retroflexus $\mathrm{L}$.

\section{HABITAT}

yards, fields, shelterbelts gardens, fields, roadsides

hillsides, prairie (burned sites)

dry prairie, heavy soils

farmyards, waste places

saline flats, slough margins

roadsides, fields, waste places

saline flats

\section{Purslane Family: Portulacaceae}

Purslane Portulaca oleracea L.

gardens, introduced weed

Pink Family: Caryophyllaceae

Field Chickweed

Mouse-eared Chickweed

Annual Baby's-breath

Baby's-breath

Cow Cockle

Night-flowering Catchfly

Salt-marsh Sand Spurry

Fleshy Stitchwort

Long-stalked Stitchwort
Cerastium arvense $\mathrm{L}$.

Cerastium vulgatum $\mathrm{L}$.

Gypsophila elegans Bieb.

Gypsophila paniculata L.

Saponaria officinalis L.

Silene noctiflora $\mathrm{L}$.

Spergularia marina (L.) Griseb. saline shores

Stellaria crassifolia Ehrh.

Stellaria longipes Goldie prairie

pasture, woodlands, fields

picnic site, roads, escaped farmsite, roadsides, escaped farmsteads, grainfields

fields, introduced

bog area

moist areas, native prairie

\section{Crowfoot Family: Ranunculaceae}

Canada Anemone

Long-fruited Anemone

Crocus Anemone

Tall Buttercup

Large-leaved Watercrowfoot

Seaside Buttercup

Shiny-leaved Buttercup

Macoun's Buttercup

Prairie Buttercup

Veiny Meadow-rue
Anemone canadensis $\mathrm{L}$.

Anemone cylindrica A. Gray

Anemone patens L.

Ranunculus acris L.

Ranunculus aquatilis L.

Ranunculus cymbalaria Pursh saline areas, sloughs, banks

Ranunculus glaberrimus Hook grazed pasture near stockpond Ranunculus macounii Britt.

Ranunculus rhomboideus

Goldie

Thalictrum venulosum Trel. low moist prairie, near bluffs moist prairie

native prairie, heavily grazed wetland ditches, moist areas dugout (not saline)

along road, moist places

ungrazed pasture, prairie woodlands, damp low prairie

Poppy Family: Papaveraceae

Corn Poppy

Papaver rhoeas L.

farmstead, introduced

Fumitory Family: Fumariaceae

Golden Corydalis

Corydalis aurea Willd.

moist areas, beaches

\section{Caper Family: Capparidaceae}

Bee Plant/Spiderflower

Cleome serrulata Pursh

sandy soil, roadsides

Mustard Family: Cruciferae

Purple Rock Cress

Hirsute Rock Cress

Reflexed Rockcress

Arabis divaricarpa A. Nels.

Arabis hirsuta (L.) Scop.

native pasture

prairie, light loam

Arabis holboellii Hornem. var. sandy native prairie retrofracta (Graham) Rydb. 
COMMON NAME

Shepherd's Purse

Gray Tansy Mustard

Flixweed

Yellow Whitlow-grass

Creeping Whitlow-grass

Dog mustard

Small-flowered Prairie-rocket

Dame's-rocket

Common Pepper-grass

Sand Bladderpod

Marsh Yellow Cress

Tumbling Mustard

Mouse-ear Cress

Stinkweed

\section{Saxifrage Family: Saxifragaceae}

Alumroot

Northern Grass-of-Parnassus

Wild Black Currant

Golden Currant

Northern Gooseberry

\section{Rose Family: Rosaceae}

Saskatoon

Hawthorn

Smooth Wild Strawberry

Three-flowered Avens

Silverweed

White Cinquefoil

Graceful Cinquefoil

Wooly Cinquefoil

Rough Cinquefoil

Bushy Cinquefoil

Prairie Cinquefoil

Low Cinquefoil

Pin Cherry

Chokecherry

Low Prairie Rose

Wood's Rose

Narrow-leaved Meadowswee

\section{Legume Family: Leguminosae}

Purple Milk-vetch

Two-grooved Milk-vetch

Canadian Milk-vetch

Ground-plum
SPECIES

Capsella bursa-pastoris (L.)

Medic.

Descurainia richardsonii

(Sweet) O.E. Schulz

Descurainia sophia (L.) Webb. disturbed areas, introduced

Draba nemorosa L.

Draba reptans (Lam.)

Erucastrum gallicum

(Willd.)O.E. Schulz

Erysimum inconspicuum

(S.Wats.) MacM.

Hesperis matronalis L.

Lesquerella arenosa (Richards) grazed pasture, dry native

Rydb. var. arenosa

(Richards) Wats.

Rorippa islandica (Oeder)

Borbas

Sisymbrium altissimum L.

Thellungiella salsuginea (Pall.)

O.E. Schulz

Thlaspi arvense L.

Heuchera richardsonii R.Br.

Parnassia palustris L.

Ribes americanum Mill.

Ribes aureum Pursh

Ribes oxyacanthoides L.

Amelanchier alnifolia Nutt.

Crataegus chrysocarpa Ashe

Fragaria virginiana Dcne.

Geum triflorum Pursh

Potentilla anserina L.

Potentilla arguta Pursh

Potentilla gracilis Dougl. var.

flabelliformis (Lehm.) Nutt.

Potentilla hippiana Lehm.

Potentilla norvegica L.

Potentilla paradoxa Nutt.

Potentilla pensy/vanica L.

Potentilla plattensis Nutt.

Prunus pensylvanica L.F.

Prunus virginiana $L$.

Rosa arkansana Porter

Rosa woodsii Lindl.

Spiraea alba Du Roi

Astragalus agrestis Dougl.

Astragalus bisulcatus (Hook.)

Gray

Astragalus canadensis L.

Astragalus crassicarpus Nutt.
HABITAT

gardens, waste places, fields, introduced

fields, waste places

grazed pasture

sandy prairie

pasture, introduced

dry, sandy prairie

escaped, yards, hedges

grassland

lakeshore, slough edge, wet places

cultivated fields, gardens

saline prairie

fields, waste place

native prairie, depressions

moist hummocky area

bluffs, coulees, on islands

sandy peninsula

bluffs, coulees

coulees, bluffs

coulees, slopes, depressions

moist, shady areas

open prairie

along streams, lakeshore

moist meadows, slough edges

moist grassland, wet meadows

dry native prairie

meadows, waste areas, introd. moist sandy beach

moist grasslands

dry native prairie

near bluffs, coulees

near bluffs, coulees

native prairie, sandy soils

bluffs, coulees on prairies

depressions in native prairie

shade of willows in prairie

sandy prairie, along beach

moist native prairie

native prairie 
COMMON NAME

Slender Milk-vetch

Missouri Milk-vetch

Narrow-leaved Milk-vetch

Racemose Milk-vetch

Ascending Milk-vetch

Loose-flowered Milk-vetch

Common Caragana

Wild Licorice

Alfalfa

White Sweet-clover

Yellow Sweet-clover

Late Yellow Locoweed

Reflexed Locoweed

Early Yellow Locoweed

Showy Locoweed

White Prairie-clover

Purple Prairie-clover

Silverleaf Psoralea

Golden-bean

Alsike Clover

American Vetch

Tufted Vetch

\section{SPECIES}

Astragalus flexuosus Dougl.

Astragalus missouriensis Nutt.

Astragalus pectinatus Dougl.

Astragalus racemosus Pursh

Astragalus striatus Nutt.

Astragalus tenellus Pursh

Caragana arborescens Lam.

Glycyrrhiza lepidota (Nutt.)

Pursh

Medicago sativa L.

Melilotus alba Medic.

Melilotus officinalis (L.) Pall.

Oxytropis campestris (L.) D.C. dry prairie

Oxytropis deflexa (Pall.) D.C. old fields

Oxytropis sericea Nutt.

Oxytropis splendens Dougl.

Petalostemon candidum

(Willd.) Michx.

Petalostemon purpureum

(Vent.) Rydb.

Psoralea argophylla Pursh

Thermopsis

rhombifolia(Nutt.)Richardson

Trifolium hybridum $\mathrm{L}$.

Vicia americana Muhl.

Vicia cracca L.

\section{HABITAT}

native prairie along lakeshore slopes, eroded hillside, prairie native prairie dry prairie slopes dry grasslands, hillsides lakeshore, coulees introduced, farmsteads

near lake, slough margins hayfields, roadsides, introd. fields, ditches, introduced fields, roadsides, introduced old fields near shelterbelt native grasslands

lakeshore, dry prairie

prairie, hillsides, dry banks native prairie prairie, roadsides, disturbed areas

farmsteads, roads, introduced around bluffs, sandy beach roadside, introduced

\section{Geranium Family: Geraniaceae}

Stork's-bill

Flax Family: Linaceae

Lewis Wild Flax

Large-flowered Yellow Flax

\section{Maple Family: Aceraceae}

Box-elder

\section{Mallow Family: Malvaceae}

Small-flowered Mallow

Round-leaved Mallow

Scarlet Mallow

\section{Violet Family: Violaceae}

Early Blue Violet

Bog Violet

Nuttall's Yellow Violet

Crowfoot Violet
Erodium cicutarium (L.) L'Her. farmstead

Linum lewsii Pursh

Linum rigidum Pursh

dry prairie

sandy soils

Acer negundo L.

shelterbelts, streams, coulees

Malva parviflora $\mathrm{L}$.

Malva pusilla Sm.

Sphaeralcea coccinea (Pursh)

Rydb.

Viola adunca J.E. Smith

Viola nephrophylla Greene

Viola nuttallii Pursh

Viola pedatifida G. Don farmyards, fields, introduced old farmstead

dry prairie, disturbed areas

wet meadow, shady areas moist meadows, sloughs open prairie

prairie, damp sandy loam

Oleaster Family: Elaeagnaceae

Silverberry

Buffaloberry
Elaeagnus commutata Bernh. Shepherdia argentea Nutt. moist light soils

lakeshore, coulees, light soils 
COMMON NAME

SPECIES

\section{Evening-Primrose Family: Onagraceae}

Fireweed

Marsh Willowherb

Scarlet Gaura

Yellow Evening-primrose

White Evening-primrose
Epilobium angustifolium L.

Epilobium palustre L.

Gaura coccinea Pursh

Oenothera biennis L.

Oenothera nuttallii Sweet
HABITAT

moist places, island edge of spring pond dry native grasslands hay field sandy soil, fields, roadsides

\section{Water Milfoil Family: Haloragaceae}

Spiked Water-milfoil

Myriophyllum exalbescens

Fern.

sloughs, lake

\section{Mare's Tail Family: Hippuridaceae}

Mare's-tail

Hippuris vuglaris L.

\section{Parsley Family: Umbelliferae}

Water-Hemlock

Plains Cymopterus

Leafy Musineon

Water-Parsnip

Heart-leaved Alexanders
Cicuta maculata L.

Cymopterus acaulis (Pursh)

Raf.

Musineon divaricatum (Pursh)

Nutt.

Sium suave Walt.

Zizia aptera (Gray) Fern. sloughs, streams, banks

marshes, springs or bogs

dry prairie, hillsides

prairie grasslands wetlands, sloughs, ponds moist native prairie

\section{Dogwood Family: Cornaceae}

Red-osier Dogwood

Cornus stolonifera Michx.

coulees

\section{Primrose Family: Primulaceae}

Pygmyflower

Saline Shootingstar

Sea-Milkwort

Fringed Loosestrife

Lance-leaved Loosestrife

Mealy Primrose

\section{Olive Family: Oleaceae}

Common Lilac

Syringa vulgaris L.

Gentian Family: Gentianaceae

Oblong-leaved Gentian

Northern Gentian

Gentiana affinis Griseb.

Gentiana amarella L.
Androsace septentrionalis L. dry soils, fields

Dodecatheon pulchellum

(Raf.) Merr.

Glaux maritima L.

Lysimachia ciliata L.

Lysimachia hybrida Michx.

Primula incana M.E. Jones moist to wet saline areas moist saline areas drying slough marsh saline meadows, moist places

\section{Dogbane Family: Apocynaceae} Indian-hemp

Phlox Family: Polemoniaceae Moss Phlox

\section{Borage Family: Boraginaceae}

Large-flowered Stickseed

Bluebur

Bluebur

Narrow-leaved Pucoon
Apocynum cannabinum L. var. hypericifolium (Ait.) Gray aspen bluffs, island

Phlox hoodii Richardson

native prairie, dry sandy loam

Hackelia floribunda (Lehm.) Johnst.

Lappula echinata Gilib.

island, high ground

fields, pastures, introduced

prairie, saline moist meadow heavily grazed native prairie Lappula occidentalis (S. Wats.) 
COMMON NAME

Mint Family: Labiatae

Hemp-nettle

Western Water-horehound

Field Mint

Marsh Skullcap

Marsh Hedge-nettle

\section{Potato Family: Solanaceae}

Wild Tomato

Solanum triflorum Nutt.

Galeopsis tetrahit L.

Lycopus asper Greene

Mentha arvensis L.

Scutellaria galericulata L.

Stachys palustris L. var. pilosa (Nutt.) Fern.

\section{Figwort Family: Scrophulariaceae}

Yellow Toad-flax

Owl's-clover

White Beardtongue

Lilac-flowered Beardtongue

Smooth Blue Beardtongue

Slender Beardtongue

Marsh Speedwell

Linaria vulgaris Miller

Orthocarpus luteus Nutt.

Penstemon albidus Nutt.

Penstemon gracilis Nutt.

Penstemon nitidus Dougl.

Penstemon procerus Dougl.

Veronica scutellata L.

\section{Bladderwort Family: Lentibulariaceae}

Greater Bladderwort Utricularia vulgaris L.

\section{Broom-rape Family: Orobanchaceae}

Clustered Broom-rape

Orobanche fasciculata Nutt.

Plantain Family: Plantaginaceae
Saline Plantain
Plantago eriopoda Torr.
Common Plantain
Plantago major L.

\section{Madder Family: Rubiaceae}

Northern Bedstraw

Galium boreale L.

\section{Honeysuckle Family: Caprifoliaceae}

Western Snowberry

Symphoricarpos occidentalis Hook.

\section{Bluebell Family: Campanulaceae}

Harebell

Campanula rotundifolia L. low meadows, island

Lobelia Family: Lobeliaceae

Spiked Lobelia

Lobelia spicata Lam.

\section{Composite Family: Compositae}

Yarrow

False Dandelion

Perennial Ragweed

Low Everlasting

Small-leaved Everlasting

Leafy Arnica

Biennial Wormwood

Plains Wormwood

Pasture Sage
Achillea millefolium L. var. lanulosa

(Nutt.) Piper

Agoseris glauca (Rush) Ref. moist prairie

Ambrosia coronopifolia

T. \& G.

Antennaria aprica Greene

Antennaria parvifolia Nutt.

Arnica chamissonis Less.

Artemisia biennis Willd.

Artemisia campestris L.

Artemisia frigida Willd.

dry prairies dry sandy soil

moist, grasslands, introduced

dry prairie, saline sites

dry prairie

native grasslands

roadside, dry hillsides

moist, slough \& brush margins

slough edges, marshes

ponds, marshes

on roots of Artemisia spp.

saline or alkaline, marsh edges roadsides, yards, lawns

moist sites - prairie, bluffs

prairie, coulees, woodlands

roadsides, lakeshore

dry prairie, saline meadows

slough bottoms, moist areas slough margins, roadsides dry native prairie

prairie, overgrazed 
COMMON NAME

Prairie Sage

Rush Aster

Many-flowered Aster

Western Willow Aster

Smooth Aster

Few-flowered Aster

Nodding Thistle

Canada Thistle

Flodman's Thistle

Wavy-leaved Thistle

Scapose Hawk's-Beard

Narrow-leaved Hawk's-Beard

Rough Fleabane

Tufted Fleabane

Canada Fleabane

Smooth Fleabane

Hirsute Fleabane

Great-flowered Gaillardia

Gumweed

Broomweed

Lance-leaved Pyrrocoma

Mountain Sneezeweed

Sunflower

Beautiful Sunflower

Tuberous-rooted Sunflower

Hairy Golden-Aster

Povertyweed

False Ragweed

Blue Lettuce

Meadow Blazingstar

Dotted Blazingstar

Skeletonweed

Pineappleweed

Prairie False Dandelion

Arrow-leaved Colt's-foot

Long-headed Coneflower

Silvery Groundsel

Marsh Ragwort

Entire-leaved Groundsel

Balsam Groundsel
SPECIES

Artemisia ludoviciana Nutt.

Aster borealis (T.\& G.)

Provancher

Aster ericoides L. ssp. pansus prairie, roadsides

(Blake) A.G. Jones

Aster hesperius A. Gray

Aster laevis L.

Aster pauciflorus Nutt.

Carduus nutans $\mathrm{L}$.

Cirsium arvense (L.) Scop.

Cirsium flodmanii (Rydb.)

Arthur

Cirsium undulatum (Nutt.)

Spreng

Crepis runcinata (James)

T. \& B.

Crepis tectorum L.

Erigeron asper Nutt.

Erigeron caespitosus Nutt.

Erigeron canadensis L.

Erigeron glabellus Nutt.

Erigeron lonchophyllus Hook.

Gaillardia aristata Pursh

Grindelia squarrosa (Pursh)

Danal.

Gutierrezia sarothrae

(Pursh)Britt.\& Rusby

Haplopappus lanceolatus

(Hook.) T.\& G.

Helenium autumnale $\mathrm{L}$.

Helianthus annuus L.

Helianthus laetiflorus Pers.

Helianthus nuttallii T. \& G.

Heterotheca villosa (Pursh)

Skinners

Iva axillaris Pursh

Iva xanthifolia Nutt.

Lactuca pulchella (Pursh) DG. old fields, roadsides

Liatris ligulistylis (A. Nels.) K. moist meadows, slough

Schum.

Liatris punctata Hook.

Lygodesmia juncea (Pursh) D

Don

Matricaria matricarioides

(Less.)Porter

Microseris cuspidata (Pursh)

Schultz-Bip.

Petasites sagittatus (Pursh) A.

Gray

Ratibida columnifera

(Nutt.)Woot.\&Standl.

Senecio canus Hook.

Senecio congestus (R.Br.) DC. moist prairie, slough margins

Senecio integerrimus Nutt.

Senecio pauperculus Michx.
HABITAT

slough margins, moist prairie

mineral fen

moist areas

moist prairie around bluffs

saline prairie (rare)

disturbed areas, introduced

disturbed sites, introduced

prairie

near lake, sandy soils

moist prairie

disturbed soil

native prairie, dry hillsides

dry prairies, roadsides

old gardens, fields, dry soils native prairie

saline flats, lakeshore

arid, native prairie

dry native prairie, saline areas low meadows, slough edges roadsides

roadsides

moist prairie, slough margins

dry sandy prairie

saline flats, fields

roadsides, vacant land

margins

dry prairie hillsides

dry native prairie, sandy soils

farmsteads, introduced

native prairie

partial shade, slough margins

old fields, roadsides, dry

prairie

dry prairie

native prairie

moist prairie, sandy soils 
COMMON NAME

Graceful Goldenrod

Low Goldenrod

Velvety Goldenrod

Showy Goldenrod

Upland White Goldenrod

Stiff Goldenrod

Mountain Goldenrod

Perennial Sow-thistle

Red-seeded Dandelion

Dandelion

Goat's-beard

\section{SPECIES}

Solidago canadensis L. var. gilvocanescens Rydb.

Solidago missouriensis Nutt. Solidago mollis Bartl.

Solidago nemoralis Ait.

Solidago ptarmicoides (Nees)

Boiv.

Solidago rigida $\mathrm{L}$.

Solidago spathulata DG.

Sonchus arvensis $\mathrm{L}$.

Taraxacum laevigatum (Willd.)

DC.

Taraxacum officinale Weber

Tragopogon dubius Scop.
HABITAT

native prairie

dry prairie

dry prairie, roadsides

sandy soils, prairie

dry prairie (rare)

dry prairie

ditch sides, grasslands

roadsides, fields, moist soils

waste places, introduced

pasture, roadsides, lawns

roads, field edges, introduced

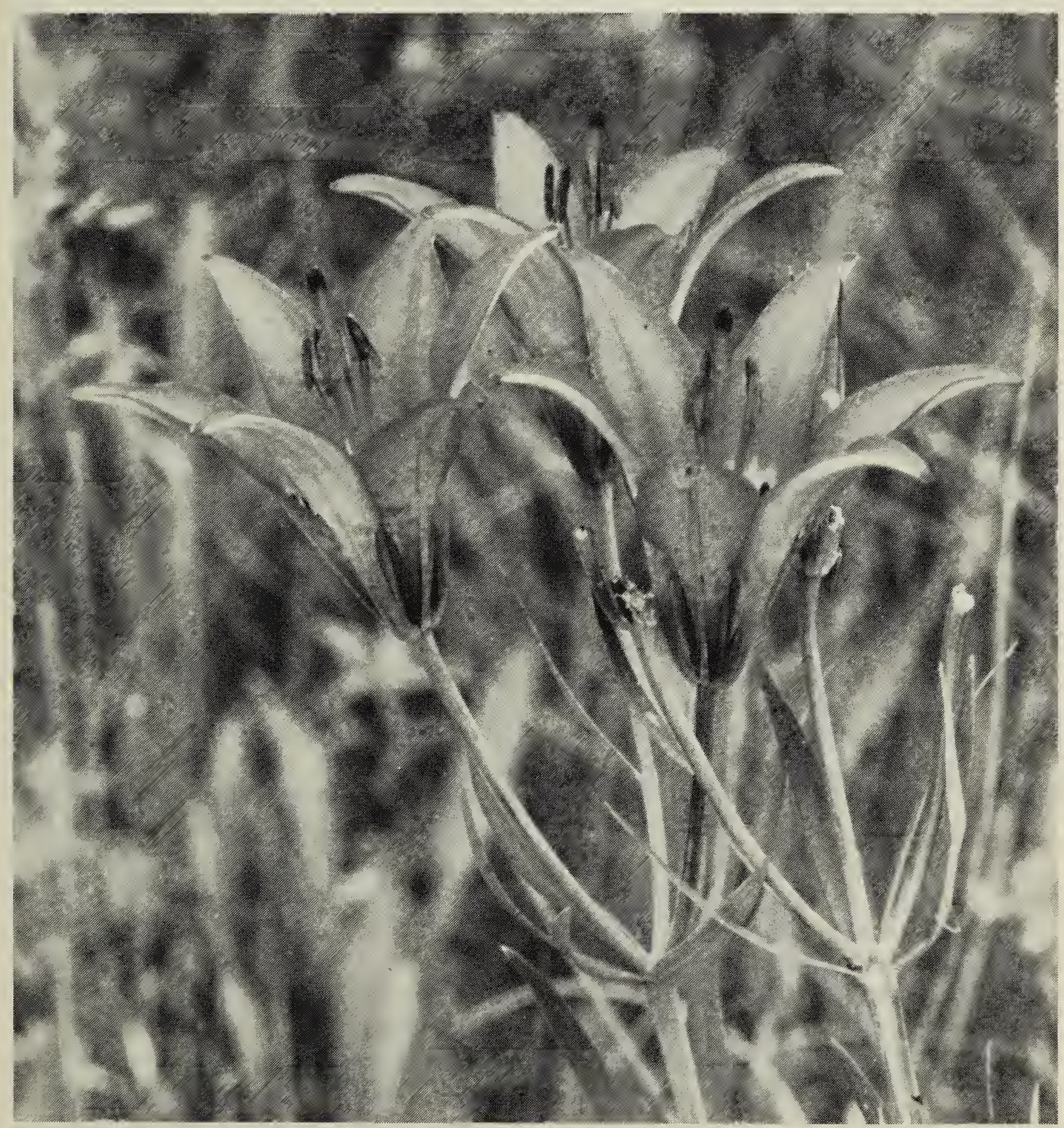

Western Wood Lily

A. Whyte 